\title{
Sunflower meal with and without phytase supplementation in diets for silver catfish (Rhamdia quelen) juveniles
}

Harina de girasol con y sin suplementación de fitasa en dietas para juveniles de bagre (barbudo, Col.; Rhamdia quelen)

Farelo de girassol com e sem suplementação de fitase em dietas para juvenis de jundiá (Rhamdia quelen)

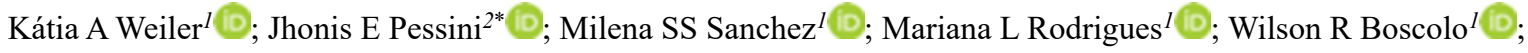

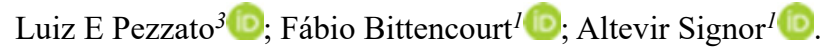

${ }^{1}$ Aquaculture Management Studies Group, Universidade Estadual do Oeste do Paraná - UNIOESTE, Toledo, Paraná, Brasil

${ }^{2}$ Aquaculture Species Nutrition Laboratory, Universidade Federal de Santa Catarina - UFSC, Florianópolis, Santa Catarina, Brasil

${ }^{3}$ College of Veterinary Medicine and Animal Science, Universidade Estadual Paulista - UNESP, Botucatu, São Paulo, Brasil

Received: July 26, 2018; accepted: April 12, 2019

To cite this article:

Weiler KA, Pessini JE, S-Sánchez MS, Rodrigues ML, Boscolo WR, Pezzato LE, Bittencourt F, Signor A. Sunflower meal with and without phytase supplementation in diets for silver catfish (Rhamdia quelen) juveniles. Rev Colomb Cienc Pecu 2019; 32(4): 285-297. DOI: https://doi.org/10.17533/udea.rccp.v32n4a04 


\begin{abstract}
Background: The cost of feed in fish farming can exceed $70 \%$ of the total costs. Thus, the feed industry have sought to formulate balanced diets including low-cost by-products. Objective: To evaluate sunflower meal as a partial substitute $(0$, 10 , and $20 \%)$ for soybean meal, with or without phytase supplementation $(1,500 \mathrm{FTU} / \mathrm{kg})$ in isoproteic $(27 \%$ crude protein (CP)) and isoenergetic diets (3,400 kcal/ED/kg of feed) for silver catfish (Rhamdia quelen) juveniles. Methods: A total of 360 fish (weight: $15.77 \pm 0.56 \mathrm{~g}$ ) were randomly distributed in 24 tanks in a completely randomized design following a factorial scheme (3x2) with 4 replicates. The fish were evaluated on the following variables after 90 days: productive performance, hematological variables, histology of intestinal tissue, bromatological composition of carcass, and composition of bone mineral matter. Results: $20 \%$ dietary inclusion of sunflower meal improved weight gain and protein efficiency. No difference was observed in the other treatments and no interactions were observed between the other factors. Conclusion: $20 \%$ sunflower meal can be included in the feed of silver catfish as a substitute for soybean meal. Phytase inclusion (1,500 FTU/kg) did not improve the nutritional value of the treatments.
\end{abstract}

Keywords: aquaculture; aquiculture; alternative feed; diet supplementation; enzyme; fish nutrition; phytase; productive performance; Rhamdia quelen; silver catfish; sunflower meal.

\title{
Resumen
}

Antecedentes: El costo de la alimentación en piscicultura pueden superar el $70 \%$ del costo total. Las industrias de alimentos para peces han intentado formular dietas balanceadas con subproductos de menor valor en el mercado para minimizar costos. Objetivo: Evaluar la harina de girasol como sucedáneo parcial $(0,10$ y $20 \%)$ de la torta de soya, suplementada o no con fitasa (1.500 FTU $/ \mathrm{kg}$ ) en dietas isoprotéicas ( $27 \% \mathrm{de}$ PB) e isoenergéticas $3400 \mathrm{kcal} / \mathrm{ED} / \mathrm{kg}$ de ración) para bagre (barbudo, Col.; Rhamdia quelen). Métodos: Los peces, 360 juveniles $(15,77 \pm 0,56 \mathrm{~g})$, se distribuyeron aleatoriamente en 24 unidades experimentales, en un diseño completamente al azar, en esquema factorial $(3 \times 2)$ y 4 repeticiones. A los 90 días se evaluó: desempeño productivo, variables hematológicas, histología de tejido intestinal y composición bromatológica de la carcasa y de la materia mineral de los huesos. Resultados: La inclusion de $20 \%$ de harina de girasol mejoró la ganancia de peso de los peces y la tasa de eficiencia proteica de la ración, sin diferencia para las otras evaluaciones ni interacción entre los demás factores evaluados. Conclusión: La harina de girasol se puede utilizar en un $20 \%$ de la ración del bagre (barbudo, Col.) como sucedáneo a la harina de soja. La inclusión de fitasa (1.500 FTU/kg) no mejoró el valor nutritivo de estas raciones.

Palabras clave: acuacultura; acuicultura; bagre; barbudo; enzima; fitasa; harina de girasol; ingrediente alternativo; nutrición de peces; Rhamdia quelen; rendimiento productivo; suplementación dietaria; torta de girasol.

\section{Resumo}

Antecedentes: Os custos relativos a alimentação na piscicultura pode ultrapassar $70 \%$ do custo final, neste sentido, as indústrias de rações têm procurado formular dietas balanceadas com subprodutos de menor valor de mercado para minimizar estes custos. Objetivo: Esse estudo objetivou avaliar o farelo de girassol como sucedâneo parcial $(0,10$ e $20 \%)$ do farelo de soja, suplementado ou não com a fitase (1.500 FTU/kg), em dietas isoprotéicas $(27 \%$ de PB) e isoenergéticas ( $3400 \mathrm{kcal} / \mathrm{ED} / \mathrm{kg}$ de ração) para o jundiá (Rhamdia quelen). Métodos: Os peixes, 360 juvenis $(15,77 \pm 0,56 \mathrm{~g})$ foram distribuídos aleatoriamente em 24 unidades experimentais, num delineamento inteiramente casualizado, em esquema fatorial (3x2) e 4 repetições. Após 90 dias foram avaliados: desempenho produtivo, variáveis hematológicas, histologia de tecido intestinal, composição bromatológica da carcaça e de matéria mineral dos ossos. Resultados: O emprego de $20 \%$ de farelo de girassol melhorou o ganho de peso dos peixes e a taxa de eficiência protéica da ração, sem diferença nas outras avaliações, ou para a interação entre os demais fatores avaliados. Conclusão: Pode-se concluir que o farelo de girassol pode ser utilizado em $20 \%$ da ração do jundiá, como sucedâneo ao farelo de soja e, que a inclusão de fitase (1,500 FTU/kg) não melhorou o valor nutritivo destas rações.

Palavras chave: aquicultura; enzima; desempenho produtivo; farelo de girassol; fitase; ingrediente alternativo; jundiá; nutrição dos peixes; piscicultura; Rhamdia quelen. 


\section{Introduction}

The rapid growth of industrial fish production in Brazil has motivated research focused on lowering production costs and reducing environmental impact. Feed is the highest cost in intensive fish farms, being the protein source the most costly ingredient of the diets (Munguti et al., 2012; Kim et al., 2019). Therefore, the feed industry has sought to obtain balanced diets with minimized costs and maximized quality to meet fish farmers expectations (Sanchez et al., 2016).

The fish feed industry formulates diets according to the dietary habits of fish to meet its nutritional requirements at a low cost (Pezzato et al., 2009). Feed manufacturers seek protein concentrates such as cotton, peanut, and sunflower meal to replace conventional animal meal and soybean meal. Sunflower meal is a byproduct of sunflower seed oil extraction. Sunflower is one of the world's most important oilseeds (FAO, 2012). Sunflower meal has high protein value, making it an ingredient that can be used in diet formulations (Shchekoldina and Aider, 2014). It has been tested in Oreochromis niloticus (Lira, 2014), Salvelinus alpinus (Smith et al., 2018), Cyprinus carpio (Rahmdel et al., 2018), and other species.

However, feeds of plant origin may contain antinutritional substances that decrease diet digestibility and can cause lesions in the gastrointestinal tract. This can reflect in poor growth, which has limited its incorporation in diets for monogastric animals (Hussain et al., 2015; Biswas et al., 2017). Phytate (phytic acid) is among these antinutritional agents. It is present in sunflower meal and decreases mineral availability, particularly phosphorus, which may impair bone mineralization and decrease protein, carbohydrate and lipid availability due to its ability to chelate phytate with other compounds (Vohra and Satyanarayan, 2002).

Mineral supplementation is necessary in diets formulated with plant-based ingredients because monogastric animals cannot synthesize phytase -the enzyme responsible for phytate hydrolysis (Fortes-Silva et al., 2011). Hence, this study evaluated sunflower meal as a partial substitute for soybean meal, with or without phytase supplementation, in diets for silver catfish (Rhamdia quelen) juveniles.

\section{Materials and Methods}

The experimental procedures were approved by the Animal Use Ethics Committee (AUEC), under protocol number 63/14, of Universidade Estadual do Oeste do Paraná (UNIOESTE). Fish spent seven days adjusting to the experimental diets prior to the 90 days experimental period. The study was conducted at Grupo de Estudos em Manejo da Aquicultura - GEMAq of UNIOESTE - Toledo Campus- PR, Brazil.

Six isoproteic $(27 \% \mathrm{CP})$ and isoenergetic (3400 kcal/ED/kg of feed) diets were formulated to meet the nutrient requirements of the fish (Freitas et al., 2011). The diets contained 0, 10 , and $20 \%$ sunflower meal with or without phytase supplementation $(1,500 \mathrm{FTU} / \mathrm{kg}$; Table 1). The ingredients were ground to $0.5 \mathrm{~mm}$ in a hammer mill. Then weighed, manually mixed, and extruded (Ex-Micro® Extruder). Pellets were dried in a forced air ventilation oven for 48 hours at $55^{\circ} \mathrm{C}$ and stored under refrigeration $\left(4^{\circ} \mathrm{C}\right)$. Phytase from Aspergillus niger (BASFNatuphos $($ ) was added to the diets according to the methodology of Rocha et al. (2008).

A completely randomized design based on a $3 \times 2$ factorial scheme was used. The first factor was the inclusion level of sunflower meal $(0$, 10 , and $20 \%$ ). The second factor was phytase supplementation ( 0 and 1,500 FTU/kg). A total of six treatments with four replicates each was obtained. Twenty-four $1 \mathrm{~m}^{3}$ tanks were placed inside a $200 \mathrm{~m}^{3}$ wooden tank for the experiments. Each experimental unit was composed of 15 fish per tank. A total of 360 silver catfish juveniles with $15.77 \pm 0.56 \mathrm{~g}$ initial weight and $12.97 \pm$ $1.66 \mathrm{~cm}$ length were used in the experiments.

Physical and chemical parameters of water were measured weekly using a multi-parameter 
probe meter (YSI Professional Plus 6050000, YSI Incorporated 1725 Brannum Lane Yellow Springs, $\mathrm{OH}, \mathrm{USA}$ ). Water parameters were: $\mathrm{pH}$ $(8.27 \pm 0.31)$, dissolved oxygen $(7.99 \pm 1.15$ $\mathrm{mg} / \mathrm{L})$, and electrical conductivity $(41.95 \pm 3.28$ $\mu \mathrm{S} / \mathrm{cm})$. Mean water temperature $(25.50 \pm 0.72$ ${ }^{\circ} \mathrm{C}$ ) was measured daily (morning and afternoon) using a mercury bulb thermometer. The mean values of the physical and chemical variables of water were within the optimal range for silver catfish cultivation (Gomes et al., 2000).
At the end of the experimental period fish were fasted for 24 hours and subsequently anesthetized with benzocaine $(100 \mathrm{mg} / \mathrm{L})$. Weight and length were measured, and the following variables were calculated: survival (S); weight gain $(\mathrm{WG})=$ final weight $(\mathrm{g})$ - initial weight $(\mathrm{g})$; specific growth rate $(\mathrm{SGR})=\{[(\mathrm{Ln}$ (final weight (g)) - Ln (initial weight (g))]/time (days) \} x100; apparent feed conversion (AFC) $=$ feed intake $(\mathrm{g}) /$ weight gain $(\mathrm{g})$; and proteic efficiency rate $(\mathrm{PER})=$ protein intake $(\mathrm{g}) /$ weight gain $(\mathrm{g})$.

Table 1. Calculated and analyzed ingredient and nutrient composition of the experimental diets.

\begin{tabular}{|c|c|c|c|c|c|c|}
\hline Inclusion level of sunflower meal & $0 \%$ & $10 \%$ & $20 \%$ & $0 \%$ & $10 \%$ & $20 \%$ \\
\hline & \multicolumn{3}{|c|}{ Without phytase } & \multicolumn{3}{|c|}{ With phytase } \\
\hline \multicolumn{7}{|l|}{ Ingredient $(\%)$} \\
\hline Soybean meal $(42 \% \mathrm{CP})$ & 47.10 & 41.05 & 35.00 & 47.10 & 41.05 & 35.00 \\
\hline Sunflower meal & 0.00 & 10.00 & 20.00 & 0.00 & 10.00 & 20.00 \\
\hline Corn - meal & 35.15 & 30.66 & 26.16 & 35.15 & 30.66 & 26.16 \\
\hline Fish meal & 15.00 & 15.00 & 15.00 & 15.00 & 15.00 & 15.00 \\
\hline Mineral and vitamin supplement ${ }^{l}$ & 0.75 & 0.75 & 0.75 & 0.75 & 0.75 & 0.75 \\
\hline L-threonine & 0.41 & 0.39 & 0.38 & 0.41 & 0.39 & 0.38 \\
\hline DL-methionine & 0.35 & 0.30 & 0.24 & 0.35 & 0.30 & 0.24 \\
\hline Choline chloride & 0.10 & 0.10 & 0.10 & 0.10 & 0.10 & 0.10 \\
\hline Vitamin $C^{2}$ & 0.10 & 0.10 & 0.10 & 0.10 & 0.10 & 0.10 \\
\hline Calcium propionate & 0.10 & 0.10 & 0.10 & 0.10 & 0.10 & 0.10 \\
\hline Butylhydroxy toluene & 0.02 & 0.02 & 0.02 & 0.02 & 0.02 & 0.02 \\
\hline Soy oil & 0 & 0.95 & 1.89 & 0 & 0.95 & 1.89 \\
\hline Lysine $78,5 \%^{3}$ & 0.68 & 0.34 & 0.00 & 0.68 & 0.34 & 0.00 \\
\hline Phytase (Natuphos $\left.{ }^{\circledR}\right)$ & 0.00 & 0.00 & 0.00 & 0.015 & 0.015 & 0.015 \\
\hline Common salt & 0.25 & 0.25 & 0.25 & 0.25 & 0.25 & 0.25 \\
\hline \multicolumn{7}{|c|}{ Calculated nutrients (\%) } \\
\hline Starch & 27.86 & 24.29 & 20.73 & 27.86 & 24.29 & 20.73 \\
\hline Calcium & 0.83 & 0.85 & 0.87 & 0.83 & 0.85 & 0.87 \\
\hline Digestible energy (Kcal/kg) & 3400 & 3400 & 3400 & 3400 & 3400 & 3400 \\
\hline Available phosphorus & 0.48 & 0.46 & 0.45 & 0.48 & 0.46 & 0.45 \\
\hline Total lysine & 2.58 & 2.25 & 1.93 & 2.58 & 2.25 & 1.93 \\
\hline Methionine + Cystine & 1.46 & 1.46 & 1.46 & 1.46 & 1.46 & 1.46 \\
\hline Digestible protein & 27.70 & 27.34 & 26.98 & 27.70 & 27.34 & 26.98 \\
\hline Threonine & 1.73 & 1.73 & 1.73 & 1.73 & 1.73 & 1.73 \\
\hline Tryptophan & 0.40 & 0.40 & 0.40 & 0.40 & 0.40 & 0.40 \\
\hline
\end{tabular}




\begin{tabular}{lcccccc}
\hline \multicolumn{7}{c}{ Analyzed nutrients (\%) } \\
\hline Humidity & 9.69 & 8.60 & 8.39 & 8.80 & 8.49 & 8.13 \\
Ether extract & 3.25 & 3.24 & 3.87 & 3.27 & 3.12 & 3.32 \\
Crude protein & 36.39 & 36.63 & 36.63 & 36.81 & 36.81 & 36.04 \\
Crude fiber & 5.81 & 6.64 & 6.97 & 5.75 & 6.88 & 6.47 \\
\hline
\end{tabular}

\begin{tabular}{cccc}
\hline \multicolumn{5}{c}{ Nutritional composition of sunflower meal } \\
\hline Humidity (\%) & Ether extract (\%) & Crude protein (\%) & Crude fiber (\%) \\
\hline 9.57 & 0.10 & 41.98 & 3.786 \\
\hline
\end{tabular}

${ }^{I}$ Guaranteed levels per kilogram of product: Vit. A, 1,750,000 IU; Vit. D3, 375.000 IU; Vit. E, 20.000 IU; Vit. K3, $500 \mathrm{mg}$; Vit. B1, 2,000 mg; Vit. B2, 2,500 mg; Vit. B6, 2,500 mg; Vit. B12, 5,000 mg; Folic acid, $625 \mathrm{mg}$; Calcium pantothenate, $7.500 \mathrm{mg}$; Vit. C, 37,500 mg; Biotin, $50 \mathrm{mg}$; Inositol, 12,500 mg; Niacin, 8,750 mg; Choline, 100,000 mg; Co, 50 mg; Cu, 1,250 mg; Fe, 15,000 mg; I, 100 mg; Mn, 3,750 mg; Se, 75 mg; Zn, 17,500 mg. ${ }^{2}$ Nutron -Vitamin C polyphosphate with $35.0 \%$ activity. ${ }^{3}$ Synthetic amino acids supplied by Ajinomoto do Brasil.

The hematological indexes (Ranzani-Paiva et al., 2013) were evaluated in three fish per replicate. After anesthesia with benzocaine $(100 \mathrm{mg} / \mathrm{L})$, blood was collected by puncturing the caudal vessel using syringes with needles internally moistened with 3\% EDTA. The percentage of micro-hematocrits, hemoglobin levels, and erythrocyte counts were determined in a Neubauer chamber. These data were used to calculate mean corpuscular volume $(\mathrm{MCV}=$ $\mathrm{Ht} \times 10 / \mathrm{Er}$ ) and mean corpuscular hemoglobin concentration $(\mathrm{MCHC}=\mathrm{Hb} \times 100 / \mathrm{Ht})$.

Leukocyte counts, total thrombocyte counts, and leukocyte differentiation in lymphocytes, neutrophils, monocytes, SGC (special granulocytic cell), and immature lymphocytes (defense cells) were determined in blood extensions (Ranzani-Paiva et al., 2013). The readings were obtained by microscopy using $100 \mathrm{x}$ magnification. Leukocytes and thrombocytes were counted by the indirect method: total leukocytes $(\mu \mathrm{L})=[$ (number of leukocytes counted in the extension $x$ number of erythrocytes in the Neubauer chamber)/2000]; and total thrombocytes $(\mu \mathrm{L})$ $=[$ (number of thrombocytes counted in the extension $\times$ number of erythrocytes in the Neubauer chamber)/2000]. One hundred cells were counted for the determination of leukocyte differentiation by establishing the percentage of each cellular component of interest.
Fish were euthanized after blood collection by means of a benzocaine overdose $(200 \mathrm{mg} / \mathrm{L})$ and subsequently eviscerated for removal and weighing of the liver and visceral fat. The following somatic indexes were calculated from the liver and visceral fat weights: visceral somatic fat index $(\mathrm{VSFI} \%=[(\mathrm{visceral}$ fat weight (g)/body weight (g))] x 100, and hepatosomatic index $(\mathrm{HI} \%)=[($ liver weight $(\mathrm{g}) /$ body weight $(\mathrm{g}))] \mathrm{x} 100$.

Histological analysis of intestinal villi was performed on portions of the fish midgut. Sections were harvested and fixed in alfac solution for six hours, then washed and preserved with $70 \%$ alcohol to remove the fixative. Subsequently, these samples were dehydrated in increasing concentrations of ethanol, clarified in xylol, and embedded in paraffin to obtain histological sections. The histochemical analysis was performed on $7 \mu \mathrm{m}$ paraffin sections fixed on a slide and stained using the HE and PAS + alcian blue $(\mathrm{AB})$ technique. These samples were analyzed using a light microscope equipped with a camera; villi height and total height (distance from the apex to the beginning of the muscular layer, and from the apex to the end of the serosa, respectively), villi width, and epithelium thickness were measured.

After evisceration, carcasses were frozen to determine centesimal composition (moisture, mineral matter, crude protein, and ether extract) 
according to the AOAC (2000). Carcasses of three other fish per treatment were frozen for the determination of bone mineral composition; the submandibular bone and all bones of the vertebral column were ground and degreased with petroleum ether for the analysis of calcium, phosphorus, magnesium, manganese, and zinc retention (Furuya et al., 2001). Bone mineral concentration was assessed with nitro-perchloric digestion for quantification of calcium, copper, phosphorus, magnesium, manganese and zinc, determined by Flame Absorption Spectrometry (FAAS).

The data were analyzed by homogeneity and normality tests. When assumptions were met, data were submitted to variance analysis in a factorial scheme (Two-Way) at a $5 \%(\mathrm{P}<0.05)$ significance level to check for a possible interaction between the two factors (replacement of soybean meal with sunflower meal and enzyme supplementation). The mean comparison test was performed only for significant interactions excluding isolated effects. The Tukey's test $(\mathrm{p}<0.05)$ was used in the Statistica $7.1^{\circledR}$ program to compare means that showed significant differences.

\section{Results}

The average performance values of fish fed the experimental rations are shown in Table 2. The use of $20 \%$ sunflower meal in the diet improved $(\mathrm{P}<0.05)$ weight gain and the feed's protein efficiency rate. It should be emphasized that, at the levels tested, this diet did not show negative impacts $(\mathrm{P}>0.05)$ on feed conversion or other performance indexes.

Table 2. Growth performance of silver catfish juveniles fed the experimental diets for 90 days.

\begin{tabular}{|c|c|c|c|c|c|c|c|}
\hline \multirow{2}{*}{ Variables* } & \multirow{2}{*}{ Phytase } & \multicolumn{2}{|c|}{ Sunflower Meal (\%) } & \multirow[b]{2}{*}{20} & \multicolumn{3}{|c|}{$p$-value } \\
\hline & & 0 & 10 & & $\mathrm{~F}^{10}$ & $\mathrm{AL}^{11}$ & $\mathrm{I}^{12}$ \\
\hline \multirow[b]{2}{*}{$\mathrm{WG}^{1}(\mathrm{~g})$} & $\mathrm{A}^{8}$ & $69.60 \pm 0.94 \mathrm{AB}$ & $53.84 \pm 0.94 \mathrm{~B}$ & $71.78 \pm 3.41 \mathrm{~A}$ & \multirow[b]{2}{*}{ Ns } & \multirow[b]{2}{*}{0.018} & \multirow[b]{2}{*}{ Ns } \\
\hline & $\mathbf{P}^{9}$ & $63.58 \pm 7.73 \mathrm{AB}$ & $58.29 \pm 3.41 \mathrm{~B}$ & $\begin{array}{c}69.08 \pm \\
5.02 \mathrm{~A}\end{array}$ & & & \\
\hline \multirow{2}{*}{$\mathrm{AFC}^{2}$} & $\mathrm{~A}$ & $1.32 \pm 0.60$ & $1.53 \pm 0.57$ & $1.31 \pm 0.50$ & \multirow{2}{*}{ Ns } & \multirow{2}{*}{ Ns } & \multirow{2}{*}{$\mathrm{Ns}$} \\
\hline & $\mathrm{P}$ & $1.29 \pm 0.23$ & $1.47 \pm 0.52$ & $1.41 \pm 0.20$ & & & \\
\hline \multirow{2}{*}{$\operatorname{SGR}^{3}(\%)$} & $\mathrm{A}$ & $1.52 \pm 0.18$ & $1.43 \pm 0.16$ & $1.66 \pm 0.08$ & \multirow{2}{*}{ Ns } & \multirow{2}{*}{ Ns } & \multirow{2}{*}{$\mathrm{Ns}$} \\
\hline & $\mathrm{P}$ & $1.47 \pm 0.12$ & $1.30 \pm 0.34$ & $1.62 \pm 0.12$ & & & \\
\hline \multirow{2}{*}{$\mathrm{PER}^{4}(\%)$} & $\mathrm{A}$ & $3.37 \pm 0.17 \mathrm{~B}$ & $3.00 \pm 0.31 \mathrm{~B}$ & $4.21 \pm 0.25 \mathrm{~A}$ & \multirow{2}{*}{ Ns } & \multirow{2}{*}{0.01} & \multirow{2}{*}{$\mathrm{Ns}$} \\
\hline & $\mathrm{P}$ & $3.22 \pm 0.22 \mathrm{~B}$ & $2.64 \pm 0.11 \mathrm{~B}$ & $3.67 \pm 0.16 \mathrm{~A}$ & & & \\
\hline \multirow{2}{*}{$\mathrm{HI}^{5}(\%)$} & $\mathrm{A}$ & $2.03 \pm 0.36$ & $1.66 \pm 0.60$ & $1.65 \pm 0.64$ & \multirow{2}{*}{ Ns } & \multirow{2}{*}{ Ns } & \multirow{2}{*}{ Ns } \\
\hline & $\mathrm{P}$ & $2.02 \pm 0.74$ & $1.78 \pm 0.43$ & $1.49 \pm 0.63$ & & & \\
\hline \multirow{2}{*}{$\operatorname{VSFI}^{6}(\%)$} & $\mathrm{A}$ & $0.91 \pm 0.53$ & $0.92 \pm 0.44$ & $0.96 \pm 0.74$ & \multirow{2}{*}{ Ns } & \multirow{2}{*}{ Ns } & \multirow{2}{*}{$\mathrm{Ns}$} \\
\hline & $\mathrm{P}$ & $1.60 \pm 1.12$ & $1.36 \pm 1.01$ & $1.24 \pm 0.85$ & & & \\
\hline \multirow{2}{*}{$\mathrm{S}^{7}(\%)$} & $\mathrm{A}$ & $83.33 \pm 11.11$ & $94.44 \pm 3.70$ & $94.44 \pm 3.70$ & \multirow{2}{*}{ Ns } & \multirow{2}{*}{ Ns } & \multirow{2}{*}{ Ns } \\
\hline & $P$ & $75.00 \pm 5.55$ & $77.77 \pm 12.96$ & $97.22 \pm 3.70$ & & & \\
\hline
\end{tabular}

*The Tukey's test was performed only for significant interactions. ${ }^{l}$ Weight gain; ${ }^{2}$ Apparent food conversion; ${ }^{3}$ Specific growth rate; ${ }^{4}$ Protein efficiency rate; ${ }^{5}$ Hepatosomatic index; ${ }^{6}$ Viscero-somatic fat index; ${ }^{7}$ Survival; ${ }^{8} \mathrm{Absent} ;{ }^{9} \mathrm{Present}$; ${ }^{10}$ Phytase; ${ }^{l 1}$ Feed; ${ }^{12}$ Interaction. 
The histological measurements $(\mu \mathrm{m})$ of villi height and width and epithelium thickness after 90 days. oftreatmentare shownin Table 3. Thehistological analysis results showed no alterations $(\mathrm{P}>0.05)$ of the intestinal epithelium

Table 3. Histological measurements $(\mu \mathrm{m})$ villi height and width and epithelium thickness of the intestinal tissue of silver catfish juveniles fed the experimental diets for 90 days.

\begin{tabular}{|c|c|c|c|c|c|c|c|}
\hline \multirow{2}{*}{ Variables* } & \multirow{2}{*}{ Phytase } & \multicolumn{3}{|c|}{ Sunflower Meal (\%) } & \multicolumn{3}{|c|}{$p$-value } \\
\hline & & 0 & 10 & 20 & $\mathrm{~F}^{3}$ & $\mathrm{AL}^{4}$ & $\mathrm{I}^{5}$ \\
\hline \multirow{2}{*}{ Villus height $(\mu \mathrm{m})$} & $\mathrm{A}^{1}$ & 224.5352 .07 & 243.1160 .93 & 237.5285 .10 & \multirow{2}{*}{ Ns } & \multirow{2}{*}{ Ns } & \multirow{2}{*}{ Ns } \\
\hline & $\mathrm{P}^{2}$ & 239.8260 .07 & 249.2643 .13 & 253.9478 .87 & & & \\
\hline \multirow{2}{*}{ Villus width $(\mu \mathrm{m})$} & $\mathrm{A}$ & 38.6114 .39 & 38.458 .36 & 36.755 .33 & \multirow{2}{*}{ Ns } & \multirow{2}{*}{ Ns } & \multirow{2}{*}{ Ns } \\
\hline & $\mathrm{P}$ & 45.268 .36 & 42.5411 .19 & 33.279 .05 & & & \\
\hline \multirow{2}{*}{ Epithelium thickness $(\mu \mathrm{m})$} & A & 35.466 .31 & 30.9411 .74 & 37.034 .78 & \multirow{2}{*}{ Ns } & \multirow{2}{*}{ Ns } & \multirow{2}{*}{ Ns } \\
\hline & $\mathrm{P}$ & 30.9413 .07 & 33.065 .70 & 40.3116 .77 & & & \\
\hline
\end{tabular}

*The Tukey's test was performed only for significant interactions. ${ }^{1}$ Abscent; ${ }^{2}$ Present; ${ }^{3}$ Phytase; ${ }^{4}$ Feed; ${ }^{5}$ Interaction.

Table 4 shows the results of hematological and biochemical parameters in fish fed with the different rations after 90 experimental days. No differences were observed in the evaluated hematological and biochemical parameters $(\mathrm{P}>0.05)$ between fish fed with rations containing soybean meal or sunflower meal, with or without phytase.

Table 4. Hematological and biochemical parameters of silver catfish juveniles fed with experimental diets for 90 days.

\begin{tabular}{|c|c|c|c|c|c|c|c|}
\hline \multirow{2}{*}{ Variable* } & \multirow{2}{*}{ Phytase } & \multicolumn{3}{|c|}{ Sunflower meal (\%) } & \multicolumn{3}{|c|}{ p-value } \\
\hline & & 0 & 10 & 20 & $\mathrm{~F}^{6}$ & $\mathrm{AL}^{7}$ & $\mathrm{I}^{8}$ \\
\hline \multirow{2}{*}{$\begin{array}{l}\text { Erythrocytes } \\
\left(\mathrm{x} 10^{6} \mu \mathrm{L}-{ }^{1}\right)\end{array}$} & $\mathrm{A}^{4}$ & $2.18 \pm 0.28$ & $2.11 \pm 0.13$ & $2.08 \pm 0.19$ & \multirow{2}{*}{ Ns } & \multirow{2}{*}{ Ns } & \multirow{2}{*}{ Ns } \\
\hline & $\mathrm{P}^{5}$ & $2.23 \pm 0.19$ & $2.12 \pm 0.34$ & $2.15 \pm 0.31$ & & & \\
\hline \multirow{2}{*}{$\begin{array}{c}\text { Hematocrit } \\
(\%)\end{array}$} & $\mathrm{A}$ & $34.28 \pm 4.46$ & $36.33 \pm 5.57$ & $34.12 \pm 4.12$ & \multirow{2}{*}{ Ns } & \multirow{2}{*}{ Ns } & \multirow{2}{*}{$\mathrm{Ns}$} \\
\hline & $\mathrm{P}$ & $34.12 \pm 2.47$ & $33.70 \pm 3.65$ & $31.71 \pm 3.5$ & & & \\
\hline \multirow{2}{*}{$\begin{array}{l}\text { Hemoglobin } \\
\qquad\left(\mathrm{g} \mathrm{dL}^{-1}\right)\end{array}$} & $\mathrm{A}$ & $6.10 \pm 0.75$ & $6.22 \pm 1.40$ & $5.60 \pm 4.05$ & \multirow{2}{*}{ Ns } & \multirow{2}{*}{ Ns } & \multirow{2}{*}{$\mathrm{Ns}$} \\
\hline & $\mathrm{P}$ & $5.82 \pm 1.14$ & $5.47 \pm 1.09$ & $4.72 \pm 1.10$ & & & \\
\hline $\mathrm{MCV}$ & $\mathrm{A}$ & $158.60 \pm 27.19$ & $173.20 \pm 33.13$ & $165.82 \pm 30.99$ & \multirow{2}{*}{ Ns } & \multirow{2}{*}{ Ns } & \multirow{2}{*}{$\mathrm{Ns}$} \\
\hline$(\mathrm{fL})^{l}$ & $\mathrm{P}$ & $153.82 \pm 19.9$ & $162.07 \pm 28.99$ & $149.30 \pm 22.19$ & & & \\
\hline $\mathrm{MCHC}$ & $\bar{A}$ & $17.88 \pm 1.69$ & $17.44 \pm 4.27$ & $16.32 \pm 1.62$ & \multirow{2}{*}{ Ns } & \multirow{2}{*}{ Ns } & \multirow{2}{*}{$\mathrm{Ns}$} \\
\hline$(\%)^{2}$ & $\mathrm{P}$ & $17.08 \pm 3.17$ & $16.29 \pm 3.09$ & $14.90 \pm 3.23$ & & & \\
\hline Leucocytes & $\mathrm{A}$ & $45733.23 \pm 10249$ & $39732.02 \pm 19690$ & $39211.13 \pm 15501$ & \multirow[b]{2}{*}{ Ns } & \multirow[b]{2}{*}{ Ns } & \multirow[b]{2}{*}{ Ns } \\
\hline$\left(\mu \mathrm{L}^{-1}\right)$ & $\mathrm{P}$ & $43319.06 \pm 11201$ & $39013.59 \pm 15545$ & $44680.18 \pm 15679$ & & & \\
\hline
\end{tabular}




\begin{tabular}{|c|c|c|c|c|c|c|c|}
\hline \multirow{2}{*}{ Variable* } & \multirow{2}{*}{ Phytase } & \multicolumn{3}{|c|}{ Sunflower meal (\%) } & \multicolumn{3}{|c|}{ p-value } \\
\hline & & $\mathbf{0}$ & 10 & 20 & $F^{6}$ & $\mathbf{A L}^{7}$ & $\mathbf{I}^{8}$ \\
\hline \multirow{2}{*}{$\begin{array}{c}\text { Trombocytes } \\
\qquad\left(\mu \mathrm{L}^{-1}\right)\end{array}$} & A & $55375.10 \pm 19702$ & $58519.21 \pm 15172$ & $68195.78 \pm 32126$ & \multirow{2}{*}{ Ns } & \multirow{2}{*}{ Ns } & \multirow{2}{*}{ Ns } \\
\hline & $\mathrm{P}$ & $62373.10 \pm 11554$ & $64021.90 \pm 27862$ & $70144.45 \pm 23246$ & & & \\
\hline \multirow{2}{*}{$\begin{array}{c}\text { Lymphocytes } \\
(\%)\end{array}$} & $\mathrm{A}$ & $80.67 \pm 6.63$ & $82.00 \pm 9.50$ & $87.50 \pm 7.50$ & \multirow{2}{*}{ Ns } & \multirow{2}{*}{ Ns } & \multirow{2}{*}{ Ns } \\
\hline & $\mathrm{P}$ & $79.17 \pm 8.79$ & $89.83 \pm 7.46$ & $78.50 \pm 9.42$ & & & \\
\hline \multirow{2}{*}{$\begin{array}{c}\text { Neutrophils } \\
(\%)\end{array}$} & A & $15.00 \pm 4.39$ & $12.40 \pm 7.30$ & $5.40 \pm 4.09$ & \multirow{2}{*}{ Ns } & \multirow{2}{*}{ Ns } & \multirow{2}{*}{ Ns } \\
\hline & $\mathrm{P}$ & $15.60 \pm 6.26$ & $6.40 \pm 2.88$ & $12.0 \pm 5.33$ & & & \\
\hline \multirow{2}{*}{$\begin{array}{c}\text { Monocytes } \\
(\%)\end{array}$} & A & $2.67 \pm 1.21$ & $2.67 \pm 2.33$ & $2.33 \pm 1.50$ & \multirow{2}{*}{ Ns } & \multirow{2}{*}{ Ns } & \multirow{2}{*}{ Ns } \\
\hline & $\mathrm{P}$ & $1.17 \pm 0.75$ & $0.83 \pm 0.98$ & $4.33 \pm 2.80$ & & & \\
\hline SGC & A & $1.0 \pm 1.26$ & $0.17 \pm 0.41$ & $0.33 \pm 0.81$ & \multirow{2}{*}{ Ns } & \multirow{2}{*}{ Ns } & \multirow{2}{*}{ Ns } \\
\hline$(\%)^{3}$ & $\mathrm{P}$ & $1.33 \pm 2.33$ & $0.83 \pm 1.33$ & $0.33 \pm 0.52$ & & & \\
\hline \multirow{2}{*}{$\begin{array}{c}\text { Immature } \\
\text { Lymphocytes } \\
(\%)\end{array}$} & A & $1.00 \pm 1.26$ & $0.67 \pm 0.82$ & $2.00 \pm 3.94$ & \multirow[b]{2}{*}{ Ns } & \multirow[b]{2}{*}{ Ns } & \multirow[b]{2}{*}{ Ns } \\
\hline & $\mathrm{P}$ & $1.16 \pm 1.47$ & $0.16 \pm 0.40$ & $2.66 \pm 1.21$ & & & \\
\hline
\end{tabular}

*The Tukey's test was performed only for significant interactions. ${ }^{l} \mathrm{MCV}=$ mean corpuscular volume; ${ }^{2} \mathrm{MCHC}=$ mean corpuscular hemoglobin concentration, ${ }^{3} \mathrm{SGC}$ : Special granulocytic cells, ${ }^{4}$ Ausent, ${ }^{5}$ Present, ${ }^{6}$ Phytase, ${ }^{7}$ Feed, ${ }^{8}$ Interaction.

The centesimal composition values (natural matter) showed that fish fed the different diets

had similar values of moisture, mineral matter, crude protein, and ether extract (Table 5).

Table 5. Centesimal composition (natural matter) of silver catfish juveniles fed with experimental diets for 90 days.

\begin{tabular}{|c|c|c|c|c|c|c|c|}
\hline \multirow{2}{*}{ Variables* } & \multirow{2}{*}{ Phytase } & \multicolumn{3}{|c|}{ Sunflower Meal (\%) } & \multicolumn{3}{|c|}{$p$-value } \\
\hline & & 0 & 10 & 20 & $\mathrm{~F}^{3}$ & $\mathrm{AL}^{4}$ & $\mathrm{I}^{5}$ \\
\hline \multirow{2}{*}{ Humidity (\%) } & $\mathrm{A}^{l}$ & $71.72 \pm 1.76$ & $69.56 \pm 1.45$ & $67.98 \pm 1.41$ & \multirow{2}{*}{ Ns } & \multirow{2}{*}{$\mathrm{Ns}$} & \multirow{2}{*}{ Ns } \\
\hline & $\mathrm{P}^{2}$ & $70.08 \pm 1.59$ & $68.41 \pm 2.69$ & $69.42 \pm 1.20$ & & & \\
\hline \multirow{2}{*}{ Protein $(\%)$} & A & $18.80 \pm 1.68$ & $19.82 \pm 0.77$ & $19.49 \pm 0.79$ & \multirow{2}{*}{ Ns } & \multirow{2}{*}{ Ns } & \multirow{2}{*}{ Ns } \\
\hline & $\mathrm{P}$ & $18.94 \pm 0.58$ & $18.95 \pm 0.82$ & $18.75 \pm 0.82$ & & & \\
\hline \multirow{2}{*}{ Ether extract $(\%)$} & A & $5.83 \pm 1.75$ & $6.09 \pm 0.64$ & $7.18 \pm 1.01$ & \multirow{2}{*}{ Ns } & \multirow{2}{*}{ Ns } & \multirow{2}{*}{ Ns } \\
\hline & $\mathrm{P}$ & $7.13 \pm 0.70$ & $6.41 \pm 0.36$ & $7.53 \pm 0.24$ & & & \\
\hline \multirow{2}{*}{ Mineral matter $(\%)$} & $\mathrm{A}$ & $4.44 \pm 0.81$ & $4.02 \pm 1.28$ & $4.60 \pm 0.29$ & \multirow{2}{*}{ Ns } & \multirow{2}{*}{ Ns } & \multirow{2}{*}{ Ns } \\
\hline & $\mathrm{P}$ & $3.99 \pm 0.81$ & $4.03 \pm 0.71$ & $4.22 \pm 0.02$ & & & \\
\hline
\end{tabular}

*The Tukey's test was performed only for significant interactions. ${ }^{1}$ Abscent; ${ }^{2}$ Present; ${ }^{3}$ Phytase; ${ }^{4}$ Feed; ${ }^{5}$ Interaction.

The bone mineral composition $(\mathrm{mg} / \mathrm{g})$ of fish after the experimental period is shown in
Table 6. These results showed that the diets did not influence this parameter. 
Table 6. Bone mineral composition ( $\mathrm{mg} / \mathrm{g}$ ) of silver catfish juveniles fed the experimental rations for 90 days.

\begin{tabular}{|c|c|c|c|c|c|c|c|}
\hline \multirow{2}{*}{ Variable* } & \multirow{2}{*}{ Phytase } & \multicolumn{3}{|c|}{ Sunflower meal (\%) } & \multicolumn{3}{|c|}{$p$-value } \\
\hline & & 0 & 10 & 20 & $\mathrm{~F}^{8}$ & $\mathrm{AL}^{9}$ & $\mathrm{I}^{10}$ \\
\hline \multirow{2}{*}{$\mathrm{Ca}(\mathrm{mg} / \mathrm{g})^{l}$} & $\mathrm{~A}^{6}$ & 74.6518 .75 & 78.154 .98 & 91.536 .97 & \multirow{2}{*}{ Ns } & \multirow{2}{*}{ Ns } & \multirow{2}{*}{ Ns } \\
\hline & $\mathrm{P}^{7}$ & 80.143 .18 & 83.053 .54 & 82.7711 .15 & & & \\
\hline \multirow{2}{*}{$\mathrm{Mg}(\mathrm{mg} / \mathrm{g})^{2}$} & $\mathrm{~A}$ & 0.910 .27 & 1.000 .09 & 1.080 .04 & \multirow{2}{*}{ Ns } & \multirow{2}{*}{ Ns } & \multirow{2}{*}{ Ns } \\
\hline & $\mathrm{P}$ & 0.990 .07 & 1.070 .18 & 1.010 .13 & & & \\
\hline \multirow{2}{*}{$\mathrm{Mn}(\mathrm{mg} / \mathrm{g})^{3}$} & A & 0.090 .04 & 0.100 .03 & 0.070 .02 & \multirow{2}{*}{ Ns } & \multirow{2}{*}{ Ns } & \multirow{2}{*}{ Ns } \\
\hline & $\mathrm{P}$ & 0.120 .02 & 0.080 .03 & 0.100 .03 & & & \\
\hline \multirow{2}{*}{$\mathrm{P}(\mathrm{mg} / \mathrm{g})^{4}$} & A & 94.608 .06 & 98.657 .00 & 112.149 .58 & \multirow{2}{*}{ Ns } & \multirow{2}{*}{ Ns } & \multirow{2}{*}{ Ns } \\
\hline & $\mathrm{P}$ & 93.139 .26 & 103.770 .98 & 96.4415 .53 & & & \\
\hline \multirow{2}{*}{$\mathrm{Zn}(\mathrm{mg} / \mathrm{g})^{5}$} & $\mathrm{~A}$ & 0.060 .02 & 0.070 .01 & 0.050 .01 & \multirow{2}{*}{ Ns } & \multirow{2}{*}{ Ns } & \multirow{2}{*}{ Ns } \\
\hline & $\mathrm{P}$ & 0.070 .01 & 0.060 .02 & 0.060 .02 & & & \\
\hline
\end{tabular}

*The Tukey's test was performed only for significant interactions. ${ }^{I}$ Calcium; ${ }^{2}$ Magnesium; ${ }^{3}$ Manganese; ${ }^{4}$ Phosphorus; ${ }^{5}$ Zinc; ${ }^{6}$ Abscent; ${ }^{7}$ Present; ${ }^{8}$ Phytase; ${ }^{9}$ Feed; ${ }^{10}$ Interaction.

\section{Discussion}

The results showed that sunflower meal may be an effective supplement in diets formulated for silver catfish and that the addition of phytase does not have a positive effect $(\mathrm{P}>0.05)$ on production performance, blood parameters, carcass chemical composition, and bone composition. Soybean meal is the standard ingredient in the formulation of animal feed because of its high protein concentration. Sunflower meal has higher methionine values when compared to soybean meal $(0.91 \% \mathrm{x}$ $0.55 \%$ ); however, soybean meal has higher values for the other amino acids essential to fish (Lira, 2014; Furuya et al., 2001). The results of this study showed that even when differences in amino acid composition were considered, inclusion of $20 \%$ sunflower meal in the feed as a replacement for soybean meal did not impair growth performance. These results are in agreement with other studies using Tilapia rendalli, Salvelinus alpinus, Cyprinus carpio in which $20 \%$ sunflower meal inclusion did not affect fish performance (Olvera-Novoa et al., 2002; Smith et al., 2017; Rahmdel et al., 2018). On the other hand, fish fed $10 \%$ sunflower meal resulted in lower weight gain. These results confirm the evidence presented by Lira (2014), who verified lower performance of Nile tilapia fed diets containing 7.5 and $11.25 \%$ sunflower meal. Furthermore, fish fed $15 \%$ of this ingredient had better weight gain (Lira, 2014).

Gonçalves et al. (2004) determined the nutritive value of sunflower meal in a study with Nile tilapia and found no effect of phytase supplementation on protein digestibility, although digestible energy increased $230 \mathrm{kcal} / \mathrm{kg}$ compared to diets without phytase. The limited use of sunflower meal is due to its high fiber content and presence of anti-nutritional factors, although these are minimized during the oil extraction processing (Bergamim et al., 2013; González-Pérez \& Vereijken, 2007). Reduction in growth and worsening of feed conversion were evidenced in studies with inclusion levels higher than $20 \%$ due to proteolytic enzyme suppression caused by antinutritional factors present in sunflower meal (Lin et al., 2010; Lovatto et al., 2014; Rahmdel et al., 2018)

The inclusion of sunflower meal resulted in increased crude fiber levels. In spite of this increase, fish fed these diets showed no alterations in intestinal histology. Although considered an omnivorous species, the silver catfish has a carnivorous tendency: its intestine is short, and its protein requirement is higher 
than that of other omnivorous species (Meyer and Fracalossi, 2004). According to Rodrigues et al. (2012), silver catfish demonstrates a physiological adaptation capacity without performance impairment when fed fiberrich diets. This may explain the similarity in intestinal epithelium measurements in fish fed the different diets in the present study.

It is noteworthy that all blood hematological and biochemical values observed in the present study are in agreement with the literature. The hematocrit and hemoglobin values are up to $43 \%$ and $8.7 \mathrm{~g} \mathrm{dl}^{-1}$, respectively (Borges et al., 2004); MCV values varied from 139.0 to 241.9 fL (Tavares-Dias \& Moraes, 2004). However, according to Tavares-Dias \& Moraes (2004), the number of erythrocytes was above the reference range for the species (1.42 to 1.95) while $\mathrm{MCHC}$ was below the value observed for the species, which can vary between 22.7 and $32.9 \%$ (Tavares-Dias \& Morais, 2004). These alterations can be related to the physical and chemical characteristics of water, which are often extreme in culture environments and may alter hematological variables (Costa et al., 2004; Carvalho \& Fernandes, 2006). Fish size can also influence these parameters because fish release metabolic residues proportional to their body size (Tavares-Dias et al., 2000; Tavares-Dias et al., 2001). Nevertheless, hemoglobin present in erythrocytes is only accumulated with cell maturation, which explains the high number of erythrocytes and low CHCM (Tavares-Dias \& Moraes, 2004). Leukocytes, total thrombocytes, lymphocytes, SGC, and immature lymphocytes in fish fed the different diets were similar (Table 4). The number of total leukocytes and thrombocytes, SGC, and immature lymphocytes were within the normal ranges for the species (Tavares-Dias \& Moraes, 2004). The diets did not alter neutrophil values, which were within the normal range (from 1.8 to $30.0 \%$, according to Tavares-Dias et al., 2002), although total leukocytes (between 2,960 and 49,800 ul1), SGC (from 0 to $6 \%$ ), and lymphocyte percentages ( 78.0 to $89.83 \%)$ were higher than indicated for the species (from 58.0 to $68.1 \%$ ) (Tavares-Dias and Moraes, 2004). Inclusion of sunflower meal or phytase supplementation did not influence the monocytes values. Although blood parameters may be influenced by many factors, it should be noted that the diets did not influence these results. According to TavaresDias et al. (2002), the diet is directly correlated with responses to external stimuli or exposures in the environment. Conversely, erythrocyte parameters remained within the normal ranges indicating no dietary effects on blood variables.

Carcass composition indicates that the inclusion of up to $20 \%$ sunflower meal, independent of phytase supplementation, does not affect protein synthesis or lipid deposition, demonstrating that this ingredient can partially replace soybean meal in diets formulated for this species. These results confirm those reported by Olvera-Novoa et al. (2002) in a study with sunflower meal substituted for fish meal in the diet for Tilapia rendalli.

Bone mineral composition may vary with age, size, and reproductive stage of animals. The presence of anti-nutritional factors, such as phytic acid, which is common in plant-based foods, affect nutrient digestibility, especially that of minerals such as phosphorus, decreasing its absorption by up to 70\% (Rocha et al., 2008; Pontes et al., 2015). Phytase supplementation in the present study did not improve mineral utilization. This can be explained by the fact that phosphorus requirements for growth, nutrient utilization, and bone mineral deposition were met by the experimental diets without phytase supplementation.

In conclusion, 20\% sunflower meal can be used in diets formulated for silver catfish, and phytase addition does not have a positive effect on performance, blood parameters, carcass or bone composition.

\section{Declarations}

\section{Acknowledgments}

This study was partially funded by the 
Coordenação de Aperfeiçoamento de Pessoal de Nível Superior - Brasil (CAPES) - Finance Code 001

\section{Funding}

Chamada Pública MCTI/CNPq/MEC/Capes Ação Transversal no06/2011 - Casadinho/ Procad PROCESSO No: 552224/2011-5 and Chamada Pública 23/2012 Programa de Apoio a Núcleos Emergentes - Pronem (Acordo CNPq/ FA) Convênio N 821/2013.

\section{Conflicts of interest}

The authors declare they have no conflicts of interest with regard to the work presented in this report.

\section{Author contributions}

Kátia A Weiler: Responsible for the conception of the study, data collection and article writing; Jhonis E Pessini: data collection, writing, review and editing of the article; Milena S S Sanchez: Data collection, writing, review and editing of the article; Mariana L Rodrigues: Data collection, writing, review and editing of the article; Wilson R Boscolo: responsible for the experimental design, managed the project, review and editing of the article. Luiz E Pezzato: Responsible for the experimental design and revised and edited the article. Fábio Bittencourt: collected the data, review and editing of the article. Altevir Signor: Responsible for the experimental design, managed the project, review and editing of the article.

\section{References}

Association of Official Analytical Chemists - AOAC. Official methods of analysis of association of official analytical chemists. $18^{\text {th }}$ ed. Gaithersburg, Maryland; 2000.

Bergamin GT, Veiverberg CA, Silva LP, Alexandra-Pretto A, Siqueira LV, Radünz-Neto J. Extração de antinutrientes e aumento da qualidade nutricional dos farelos de girassol, canola e soja para alimentação de peixes. Cienc Rural 2013; 43:1878-1884. http://dx.doi. org/10.1590/S0103-84782013001000024

Biswas A, Araki H, Nakamori T, Kato K, Takii $\mathrm{K}$. Fish meal replacement by soy protein from soymilk in the diets of red sea bream (Pagrus major). Aquac Nutr 2017; 23:1379-1389. https://doi.org/10.1111/anu.12513

Borges A, Scotti LV, Siqueira D R, Jurinitz DF, Wassermann GF. Hematologic and serum biochemical values for jundiá (Rhamdia quelen). Fish Physiol Biochem 2004; 30:21-25. http://dx.doi.org/10.1007/s10695-004-5000-1

Carvalho CS, Fernades MN. Effect of temperature on copper toxicity and hematological responses in the neotropical fish Prochilodus scrofa at low and high $\mathrm{pH}$. Aquaculture 2006; 251:109-117. https://doi. org/10.1016/j.aquaculture.2005.05.018

Costa OFT, Ferreira DJS, Mendonça FLP, Fernandes MN. Susceptibility of the Amazonian fish, Colossoma macrapomum (Serrasalminae) to short-term exposure to nitrite. Aquaculture 2004; 232:627-636. https://doi.org/10.1016/ $\underline{\mathrm{S} 0044-8486(03) 00524-6}$

FAO, 2012. FAO Statistical Yearbook 2013-World Food and Agriculture. FAO. http://www.fao.org/3/i3107e/i3107e00.htm

Fortes-Silva R, Sánchez-Vázquez FJ, Martínez FJ. Effects of pretreating a plant-based diet with phytase on diet selection and nutrient utilization in European sea bass. Aquaculture, 2011; 319:417-422. https://doi.org/10.1016/j. aquaculture.2011.07.023

Freitas JMA, Sary C, Luchesi JD, Feiden A, Boscolo WR. Proteína e energia na dieta de jundiás criados em tanques-rede. Rev Bras Zootec 2011; 40:2628-2633. http://dx.doi. org/10.1590/S1516-35982011001200002

Furuya WM, Gonçalves GS, Furuya VRB, Hayashi C. Fitase na alimentação da tilápiado-Nilo (Oreochromis niloticus). Desempenho 
e digestibilidade. Rev Bras Zootec 2001; 30(3):924-929. http://dx.doi.org/10.1590/ $\underline{\text { S1516-35982001000400003 }}$

Gomes LC, Golombieski I, Gomes ARC, Baldisserotto B. Biologia do jundiá Rhamdia quelen (Teleostei, Pimelodidae). Cienc Rural 2000; 30 (1):179-185. http://dx.doi.org/10.1590/ $\underline{\mathrm{S} 0103-84782000000100029}$

GonçalvesGS,PezzatoLE, Barros MM, Kleeman GK, Rocha DF. Efeitos da Suplementação de Fitase sobre a Disponibilidade Aparente de Mg, $\mathrm{Ca}, \mathrm{Zn}, \mathrm{Cu}, \mathrm{Mn}$ e $\mathrm{Fe}$ em Alimentos Vegetais para a Tilápia-do-Nilo. Rev Bras Zootec 2004; 34 (6):2155-2163. http://dx.doi.org/10.1590/ $\underline{\text { S1516-35982005000700001 }}$

González-Pérez S, Vereijken JM, Sunflower proteins: overview of their physicochemical, structural and functional properties. J Sci Food Agric 2007; 87 (12):21732191. $\quad$ https://doi.org/10.1002/jsfa.2971

Hussain SM, Afzal M, Javid A, Hussain AI, Ali Q, Mustafa I. Efficacy of phytase supplementation on growth performance and mineral digestibility of Labeo rohita fingerlings fed on cottonseed meal based diet. Pak J Zool 2015; 47(3):699705. https://pdfs.semanticscholar.org/ab57/ ca65ab03713057973535f0a4708365500a46.pdf

Kim K, Park Y, Je H, Seong M, Damusaru JH, Kim S, Jung J, Bai S. Tuna byproducts as a fish-meal in tilapia aquaculture. Ecotoxicol Environ Saf 2019; 172:364-372. https://doi. org/10.1016/j.ecoenv.2019.01.107

Lin S, Mai K, Tan B, Liu W. Effects of four vegetable protein supplementation on growth, digestive enzyme activities and Liver functions of juvenile tilapia (Oreochromis niloticus $\times$ Oreochromis aureus). J World Aquac Soc 2010; 41:583-593.https://doi.org/10.1111/j.17497345.2010.00398.X

Lira, AD. Digestibilidade aparente de nutrientes e energia do farelo de girassol para juvenis de tilápia do Nilo. Dissertação (Dissertação em Zootecnita) - UFBA. Salvador. 2014. [Accessed April 2018]. URL: http://doczz. com.br/doc $/ 241661 /$ farelo-de-girassolna-alimenta\%C3\%A 7\%C3\%A3o-detil\%C $3 \%$ Alpia-do-nilo

Lovatto NL, Silva LP, Loureiro BB, Goulart FR, Pretto A, Speroni CS, Radunz-Neto J, Loro VL. Efeitos de dietas contendo concentrados proteicos vegetais no desempenho e atividade de enzimas digestivas de jundiá (Rhamdia quelen). Semin Cienc Agrar 2014; 35:1071-1081. https:// doi.org/10.5433/1679-0359.2014v35n2p1071

Meyer G, Fracalossi, DM. Protein requirement of jundia fingerlings, Rhamdia quelen, at two dietary energy concentrations. Aquaculture 2004; 240:331-343. https://doi.org/10.1016/j. aquaculture.2004.01.034

Munguti J, Charo-Karisa H, Opiyo MA, Ogello EO, Marijani E, Nzayisenga L, Liti D. Nutritive value and availability of commonly used feed ingredients for farmed Nile tilapia (Oreochromis niloticus L.) and African catfish (Clarias gariepinus, Burchell) in Kenya, Rwanda and Tanzania. Afr J Food Agric Nutr Dev 2012; 12:6135-6155. https://www.ajol.info/index.php/ ajfand/article/view/77095/67557

Olvera-Novoa MA, Oliveira-Castillo L, Martínez-Palacios CA. Sunflower seedmeal as a protein source in diets for Tilapia rendalli (Boulanger, 1896) fingerlings. Aquac Res 2002; 33 (3):223-229. https://doi.org/10.1046/j.13652109.2002.00666.x

Pezzato LE, Barros MM, Furuya WM. Valor nutritivo dos alimentos utilizados na formulação de rações para peixes tropicais. R Bras Zootec 2009; 38:43-51. http://dx.doi.org/10.1590/ $\underline{\text { S1516-35982009001300005 }}$

Pontes TC, Cagol L, Dutra FM, Portz L. Disponibilidade do fósforo em alimentos de origem vegetal: atuação na nutrição de peixes. Arq Ciênc Vet Zool 2015; 18 (3):199-205. http://revistas.unipar.br/index.php/veterinaria/ article/viewFile/5542/3135 
Rahmdel KJ, Noveirian HA, Falahatkar B, Lashkan AB. Effects of replacing fish meal with sunflower meal on growth performance, body composition, hematological and biochemical indices of common carp (Cyprinus carpio) fingerlings. Fish \& Aquat Life 2018; 126:121129. https://doi.org/10.2478/aopf-2018-0013

Ranzani-Paiva MJT, Pádua SB, Tavares-Dias M, Egami, MI. Técnicas de análise hematológica em peixes. Maringá, Eduem; 2013

Rocha CB, Pouey JLOF, Lopes PRS, Enke DBS, Xavier EG. Suplementação da enzima fitase e o desempenho e retenção mineral em juvenis de jundiá (Rhamdia quelen). Bol Inst Pesca 2008; 34 (1):151 - 157. https://www.pesca.sp.gov.br/ boletim/index.php/bip/article/view/780/763

Rodrigues APO, Gominho-Rosa, MDC, Cargnin-Ferreira E, De Francisco A, Fracalossi DM. Different utilization of plant sources by the omnivores jundiá catfish (Rhamdia quelen) and Nile tilapia (Oreochromis niloticus). Aquac Nut 2012; 18:65-72. https://doi.org/10.1111/j.1365$\underline{2095.2011 .00877 . x}$

Sanchez MSS, Nascimento MS, Hisano $H$. Replacement of corn by sorghum in diets for pacu juvenile. Pesqui Agropecu Bras 2016; 51 (1):1-8. http://dx.doi.org/10.1590/S0100204X2016000100001

Shchekoldina T, Aider M. Production of low chlorogenic and caffeic acid containing sunflower meal protein isolate and its use in functional wheat bread making. J Food Sci Technol 2014; 51:2331-2343. https://doi.org/10.1007/s13197-012-0780-2

Smith AA, Dumas A, Yossa R, Overturf KE, Bureau DP. Effects of soybean and sunflower meals on the growth, feed utilization, and gene expression in two Canadian strains of juvenile Arctic charr (Salvelinus alpinus). Aquaculture 2017; 481:191-201. https://doi.org/10.1016/j. aquaculture.2017.08.038
Smith AA, Dumas A, Yossa R, Overturf KE, Bureau DP. Effects of soybean meal and highprotein sunflower meal on growth performance, feed utilization, gut health and gene expression in Arctic charr (Salvelinus alpinus) at the growout stage. Aquac Nutr 2018; 24:1540- 1552. https://doi.org/10.1111/anu.12691

Tavares-Dias M, Melo JFB, Moraes G, Moraes FR. Características hematológicas de teleósteos brasileiros. IV. Variáveis do Jundiá (Rhamdia quelen) (Pimelodidae). Cienc Rural 2002; 32 (4):693-698. http://dx.doi.org/10.1590/S0103$\underline{84782002000400024}$

Tavares-Dias M, Moraes FR. Hematologia de peixes teleósteos. Ribeirão Preto: Villimpress; 2004.

Tavares-Dias M, Schalch SHC, Martins ML, Onaka EM, Moraes FR. Hematological characteristics of Brazilian teleostes. III. Parameters of the hybrid tambacu (Piaractus mesopotamicus, Holmberg, 1887 x Colossoma macropomum Cuvier, 1818) (Osteichthyes: Characidae). Rev Bras Zool 2000; 17 (4):899$926 . \quad$ http://dx.doi.org/10.1590/S0101$\underline{81752000000400002}$

Tavares-Dias M, Sandrim EFS, Moraes FR, Carneiro PCF. Physiological responses of "Tambaqui" Colossoma macropomum (Characidae) to a cute stress. Bol Inst Pesca 2001; 27 (1):43-48. https://www.pesca.sp.gov. br/boletim/index.php/bip/article/view/689

Vohra A, Satyanarayana T. Purification and characterisation of a thermostable and acidstable phytase from Pichia anomala. World J Microbiol Biotechnol 2002; 18:687-691. https://doi.org/10.1023/A:1016850121719 